\title{
Optimization of sequence alignment for simple sequence repeat regions
}

\author{
Abdulqader Jighly*, Aladdin Hamwieh and Francis C Ogbonnaya
}

\begin{abstract}
Background: Microsatellites, or simple sequence repeats (SSRs), are tandemly repeated DNA sequences, including tandem copies of specific sequences no longer than six bases, that are distributed in the genome. SSR has been used as a molecular marker because it is easy to detect and is used in a range of applications, including genetic diversity, genome mapping, and marker assisted selection. It is also very mutable because of slipping in the DNA polymerase during DNA replication. This unique mutation increases the insertion/deletion (INDELs) mutation frequency to a high ratio - more than other types of molecular markers such as single nucleotide polymorphism (SNPS).

SNPs are more frequent than INDELs. Therefore, all designed algorithms for sequence alignment fit the vast majority of the genomic sequence without considering microsatellite regions, as unique sequences that require special consideration. The old algorithm is limited in its application because there are many overlaps between different repeat units which result in false evolutionary relationships.
\end{abstract}

Findings: To overcome the limitation of the aligning algorithm when dealing with SSR loci, a new algorithm was developed using PERL script with a Tk graphical interface. This program is based on aligning sequences after determining the repeated units first, and the last SSR nucleotides positions. This results in a shifting process according to the inserted repeated unit type.

When studying the phylogenic relations before and after applying the new algorithm, many differences in the trees were obtained by increasing the SSR length and complexity. However, less distance between different linage had been observed after applying the new algorithm.

Conclusions: The new algorithm produces better estimates for aligning SSR loci because it reflects more reliable evolutionary relations between different linages. It reduces overlapping during SSR alignment, which results in a more realistic phylogenic relationship.

\section{Background}

Microsatellites, or simple sequence repeats (SSRs), are tandemly repeated DNA sequences with a period of from 1 to 6 base pairs [1]. It is sometimes referred to as a variable number of tandem repeats or VNTRs. An SSR which contains one type of repeats, is called a simple SSR (e.g. (CA) ${ }_{15}$ ) and those which have more than one type are called compound SSRs (e.g. $(\mathrm{CA})_{8}(\mathrm{CG})_{12}$ ) [2]. The repeat units are generally di-, tri- tetra- or pentanucleotides. They are commonly found in non-coding regions of the genome.

\footnotetext{
* Correspondence: a.jighly@cgiar.org
International Center for Agricultural Research in the Dry Areas (ICARDA), P.O.

* Correspondence: a.jighly@cgiar.org
International Center for Agricultural Research in the Dry Areas (ICARDA), P.O. Box 5466, Aleppo, Syria
}

SSRs are highly mutable loci [3]. In animals, observed SSR mutation rates have been of the order of $10^{-3}$ to $10^{-}$ ${ }^{4}$ for autosomal repeat loci [4,5] (Wiessenbach et al. 1992; Weber and Wong 1993). However the average of mutations in SSR loci is $10^{-2}$ in one generation [6].

Chistiakov et al. [7] suggested that two mechanisms are responsible for the high mutability in SSRs. First, motif repetition makes SSRs prone to mutation by DNA polymerase slippage during replication because of the multi-complementary sequences, and second, unequal crossing over or related processes [8-11]. The slippage rate is correlated to SSR length and this makes longer SSRs more variable than shorter ones [12,13]. However, there is no threshold length for slippage mutations [14]. The mutations that happen because of the polymerase 
slippage could be considered as special types of insertion/deletion (INDELs) mutations that usually occur when adding or erasing sequences without any substitution. Substitution is considered as another kind of mutation called single nucleotide polymorphism (SNPs). In general, SNPs occur much more frequently than INDELs [15]. But SSR replication slippage generates more genetic change in eukaryotes than do all base substitution per generation [16], so it increases the frequency of INDELs. In addition, it has been reported that the perfect SSR motifs are significantly more variable compared to imperfect repeated motifs $[17,18]$.

The power of SSR regions relies on their high abundance in the genome, codominant nature, extensive genome coverage, and high polymorphism [19]. The polymorphism of SSR depends on the differences in the numbers of repeated units between alleles at a single locus. The SSRs are used as molecular markers in a wide range of applications, such as genome mapping, marker assisted selection, gene tagging, and evolutionary and diversity studies [20] The main feature of SSRs that makes them amenable for use as molecular markers is that the flanking regions are highly conserved, allowing the use of specific PCR primers to amplify the same SSR even across different taxa [21,22].

Sequence alignment involves the identification of the correct location of INDELS that have happened since their divergence from a common precursor. The true alignment reflects the evolutionary relationships between the sequences accurately. Nevertheless, in the case of a compound SSR region, the general alignment will show many overlaps between the different units of repeats, which seem biologically incorrect because of the replication slippage mutations rate. This suggests a need to reevaluate the general alignment methods and their parameters. In this paper, we surmise that correct alignment should put the repeats separately without overlapping between them and without changing the alignment parameters. We suggest the incorporation of a simple algorithm for the shifting process of SSR loci after applying the usual alignment used in regular software.

\section{Findings}

\section{Algorithm}

In this paper, we compare our new algorithm for SSR alignment with the common alignment algorithms used in other programs. The new algorithm (Figure 1) would deal with the SSR according to the following major steps:

1- User must identify the following items:
a. Data set file
b. Repeated units
c. SSR length (first and last nucleotide)

2- Identify the sequences that do not match the first repeated unit from the beginning of the selected SSR region

3- Do this for each repeated unit

a. Put the tandem repeat in a temporary array

b. Check if the next nucleotides match the next repeated unit

c. If not, put the unmatched nucleotides in another temporary array

d. Fill the gaps to the longest sequence of the repeats in the same array

e. Merge the temporary arrays

4- Put your results instead of the SSR region.

See the additional file 1: SALT.swf. An animation describes the algorithm.

\section{Testing and Implementation}

The sequence case A contained a simple SSR with the tandem TA, which represents $15.4 \%$ of the whole sequence. After applying the alignment in the MEGA 4 alignment and our modifications, one major difference was shown clearly in the gap sites in some sequences (Figure 2). However, these differences did not reveal variations in the phylogenic tree before and after applying the new algorithm, and the whole sequence length equals $351 \mathrm{bp}$ in both cases (Figure 3).

The sequence case B contained a compound SSR with the tandem TA and CA, which represents $25.2 \%$ of the whole sequence. The length was increased from $397 \mathrm{bp}$ to $413 \mathrm{bp}$ after applying the new algorithm. However, the phylogenic trees indicated that $50 \%$ of the samples showed a similar cluster before and after the new algorithm being applied (Figure 4).

The sequence case $C$ contained a compound SSR of TA, CA, and CG tandem repeats representing 35\% of the whole sequence. Applying our new algorithm for case $C$ increased the length of the sequence from 457 bp to $478 \mathrm{bp}$. However, the comparison of the phylogenic trees before and after applying the new algorithm showed that only seven samples, $26.9 \%$ of the whole sequence, clustered similarly (Figure 5).

The sequence case D contained compound SSR (TA, CA, CG, and TG). The length of this tandem repeats represents $38 \%$ of the whole sequence. The whole sequence length was changed after the new algorithm was applied from $479 \mathrm{bp}$ to $539 \mathrm{bp}$. The cluster analysis resulted in completely different phylogenic trees before and after applying the new algorithm (Figure 6).

The overall pairwise value (PV) for cases A, B, C, and $\mathrm{D}$ before applying the new algorithm indicated that these values were increased whenever the sequence contained more repeated units (Figure 7). In contrast, the PV was decreased after the new algorithm was applied 


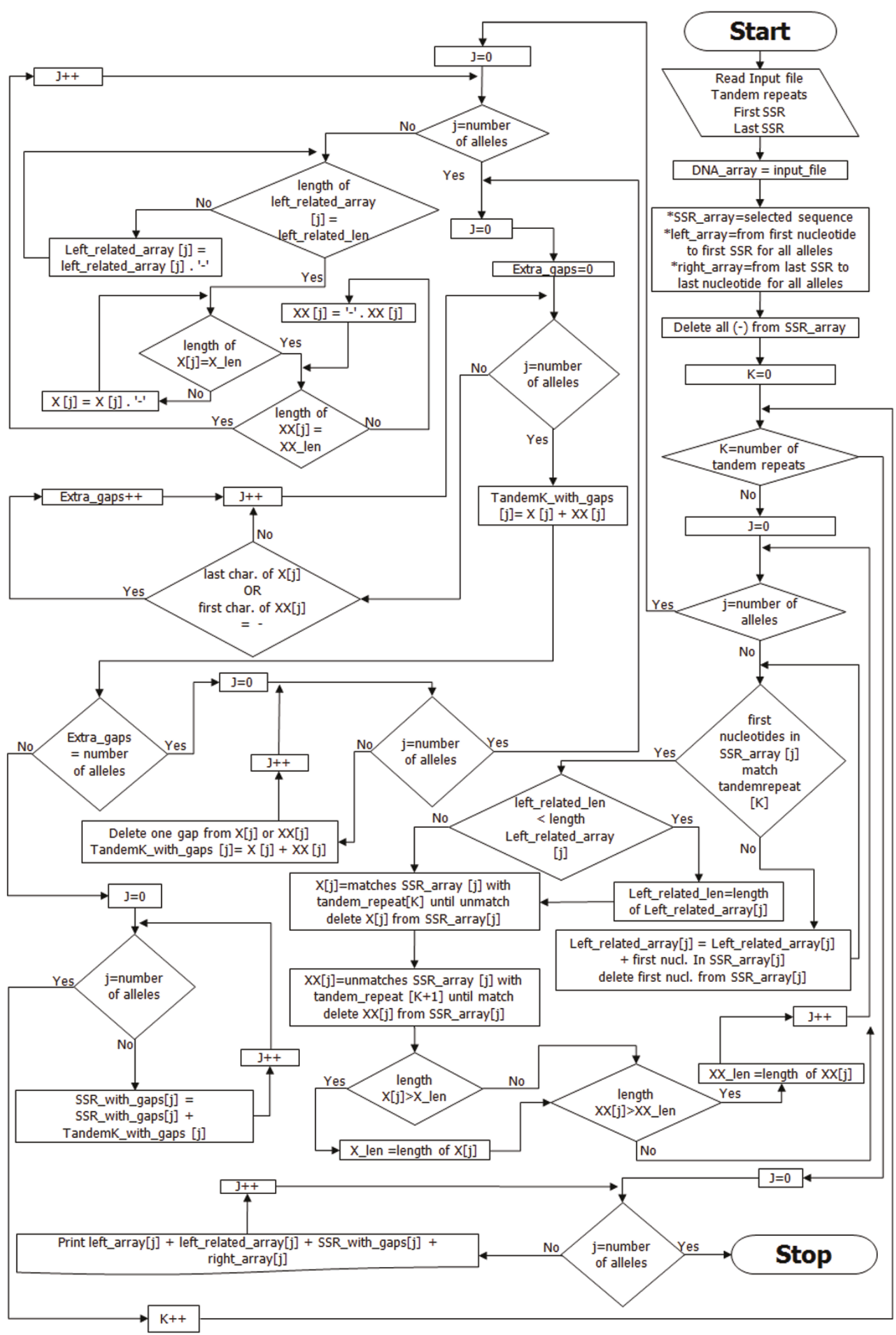

Figure 1 The SSR aligning algorithm 


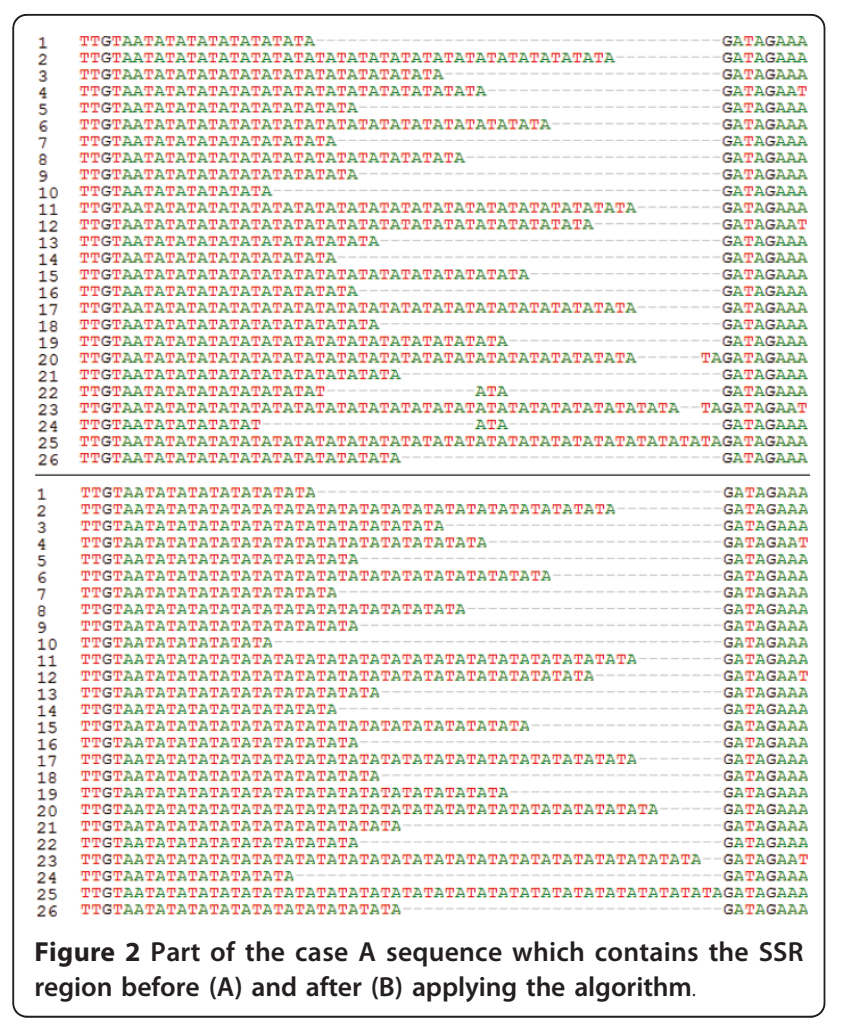

to the same sequences. Applying the new algorithm showed a more stable distance by preventing the overlaps between different linages, although it has a slight decrease, which may be attributed to the additional aligned repeated unit, The additional units increased the SSR length giving more similarity because it does not contain overlaps or mismatches and the only difference between alleles is the opening gap position. The interval values between the two PV (before and after applying the new algorithm) were increased for the cases A, B, C, and $\mathrm{D}$, indicating that the general alignment methods revealed more genetic distance.

Case E showed a compound-imperfect SSR repeat with the tandems GAA, GAT, and GAGGAT respectively. This imperfect SSR represents $9.4 \%$ of the sequence tested in case $\mathrm{E}$. The alignment process showed clear differences before and after the SSR region was treated with the new algorithm (Figure 8). Despite the small percentage of this SSR in the whole sequence in case E, the phylogenic trees showed that the genetic distance of the most 24 related sequences was decreased from 0.00317 to 0.002 (Figure 9). Further, more sequences that are similar resulted in less branches.

The main limitation with the new algorithm is in determining the gap position when applied to an

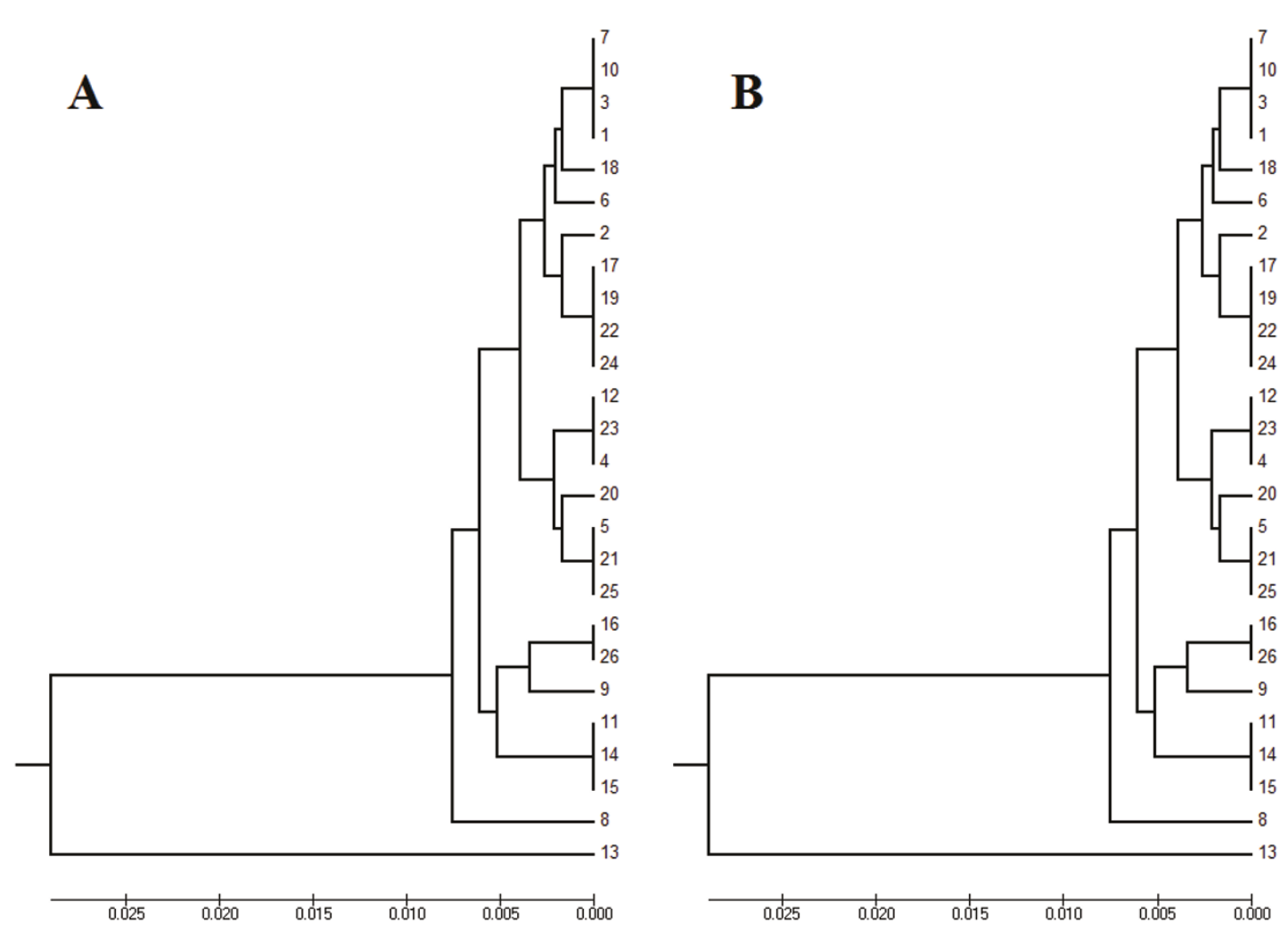

Figure 3 Case A phylogenic tree before (A) and after (B) applying the algorithm. 


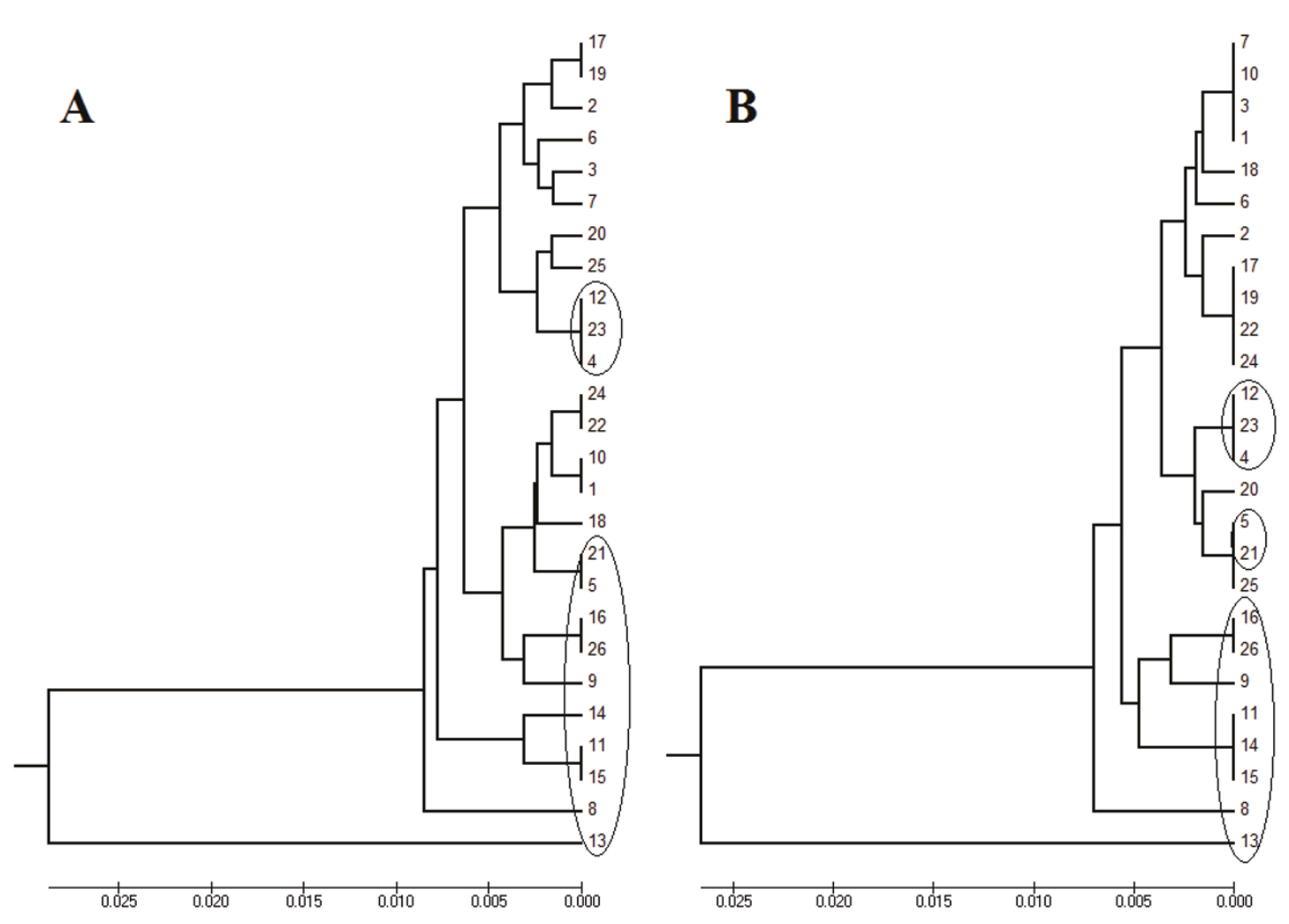

Figure 4 Case B phylogenic tree before (A) and after (B) applying the algorithm
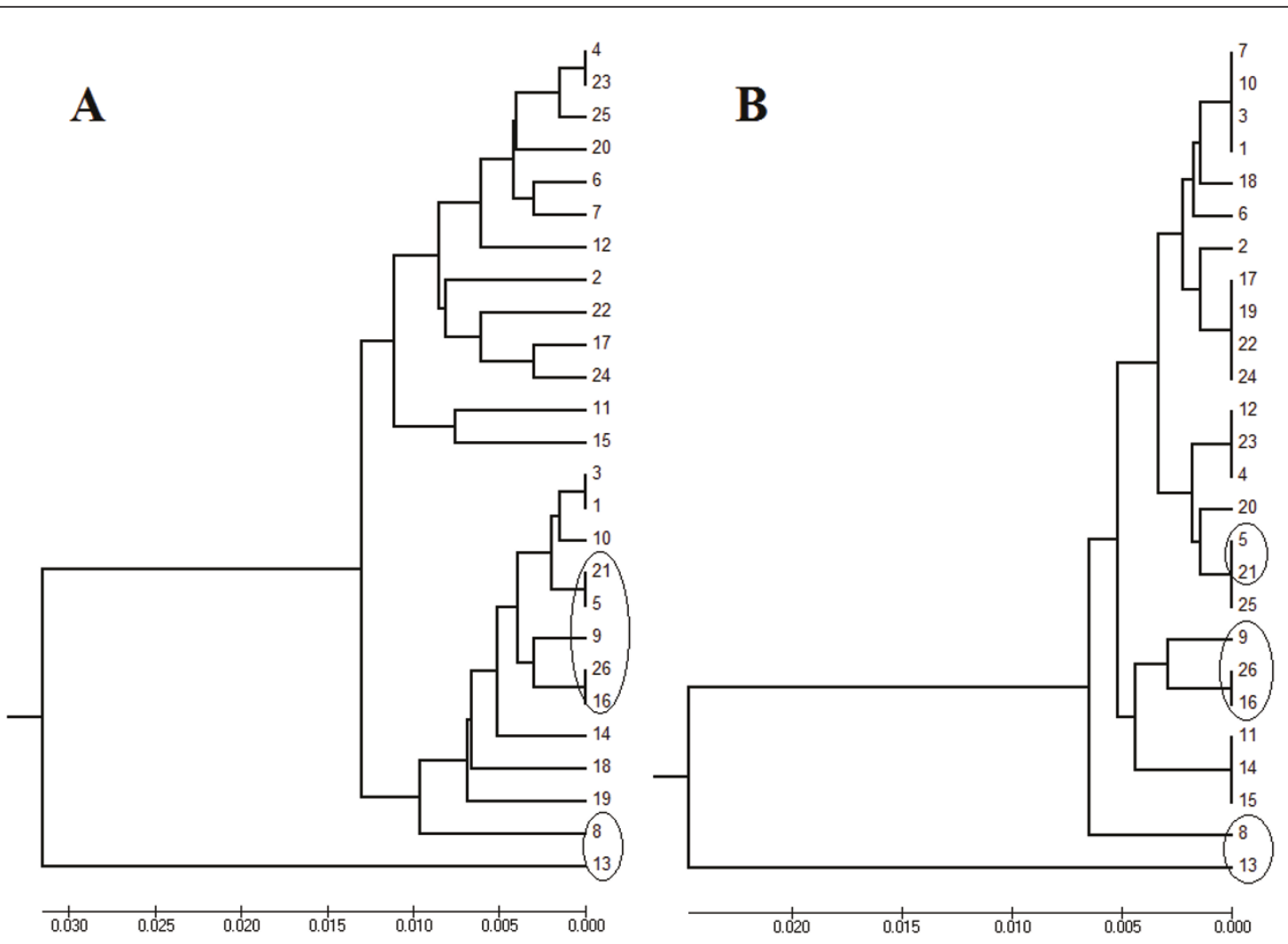

Figure 5 Case C phylogenic tree before (A) and after (B) applying the algorithm. 

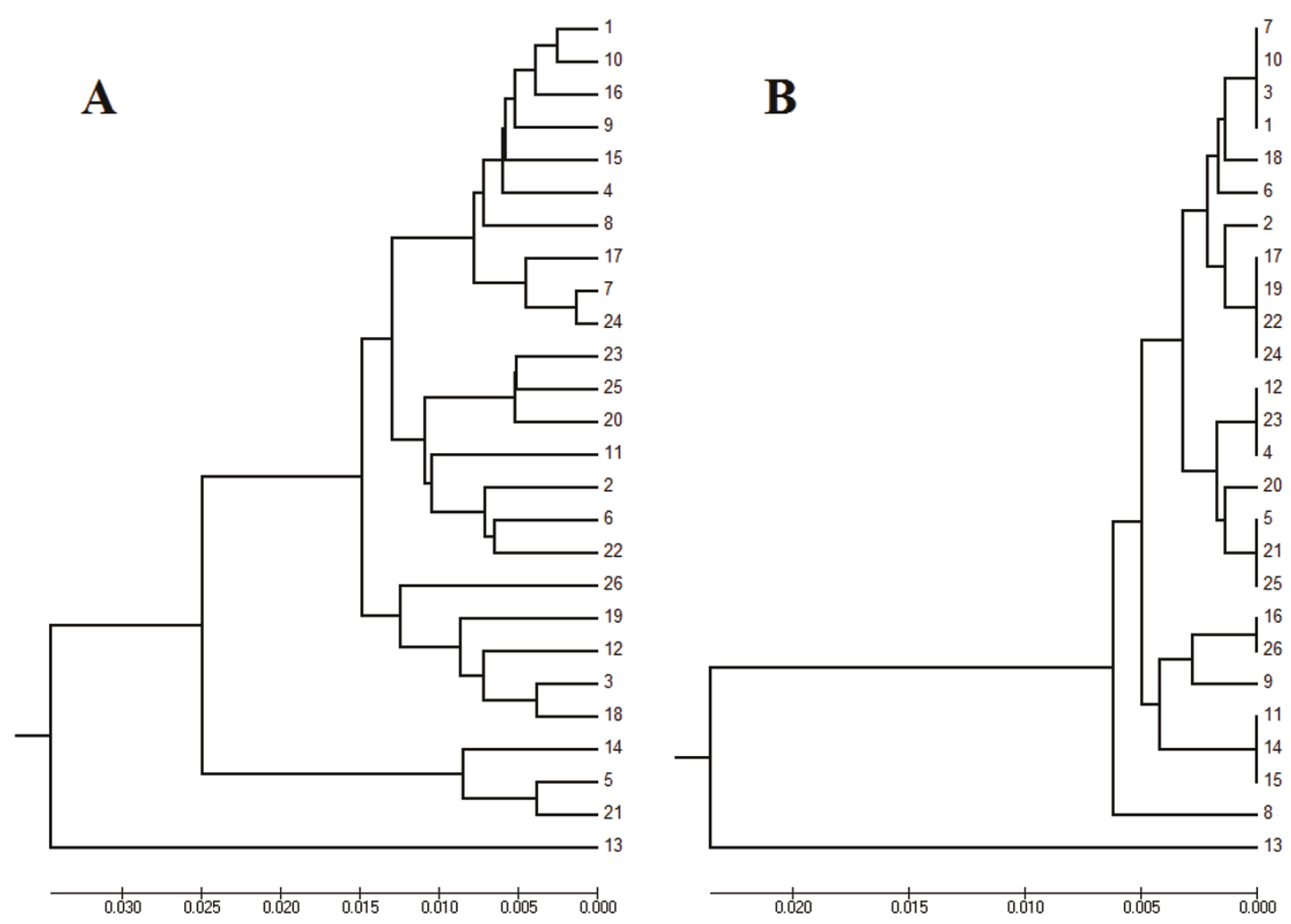

Figure 6 Case D phylogenic tree before (A) and after (B) applying the algorithm.

imperfect SSR. According to Kruglyak [17] and Bandström [18], the imperfect repeats within the SSR region reduces the occurrences of slippage, resulting in the imperfect SSR changing its tandem nature and fixing the region by prohibiting replication slippage. This is because the bases do not find their complementary bases during replication. However, the best place for the imperfect nucleotides within a compound SSR is after the slippage site (the gap) and before the sequence that follows SSR or the next repeated unit (Figure 7).

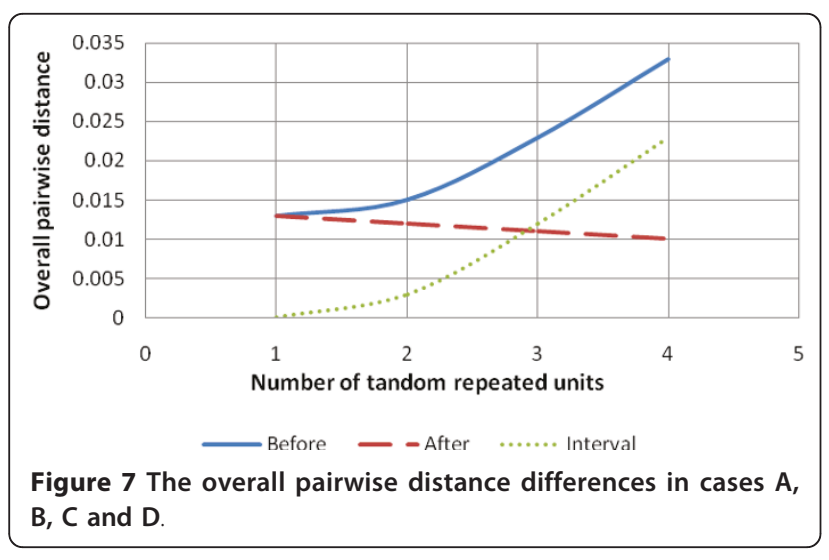

We can deduce from the last examples that (1) the new algorithm could be a powerful tool for compound SSRs, but less so for a simple SSR, (2) it increase the similarity between sequences during alignment by minimizing the overlaps between different repeated units, and (3) it might be necessary to apply it on sequences containing long and complicated SSRs.

\section{SSR alignment tool (SALT)}

SALT is a new tool for making an alignment for SSR loci using the new algorithm. It was written using the PERL programming language. Figure 10 shows the main window of the program which consists of five textboxes for the names or the directories for the input and the output files. The user should determine his tandem repeats by putting a space character between each repeated unit and the next one in the third textbox. The remaining text boxes are for identifying the first and the last nucleotide position of the SSR locus in the whole sequence. There are also four buttons, two for browsing the input and the output files, the third for making the alignment, and the last for closing the program.

The input file should be aligned sequences in fasta format or in .txt format: 


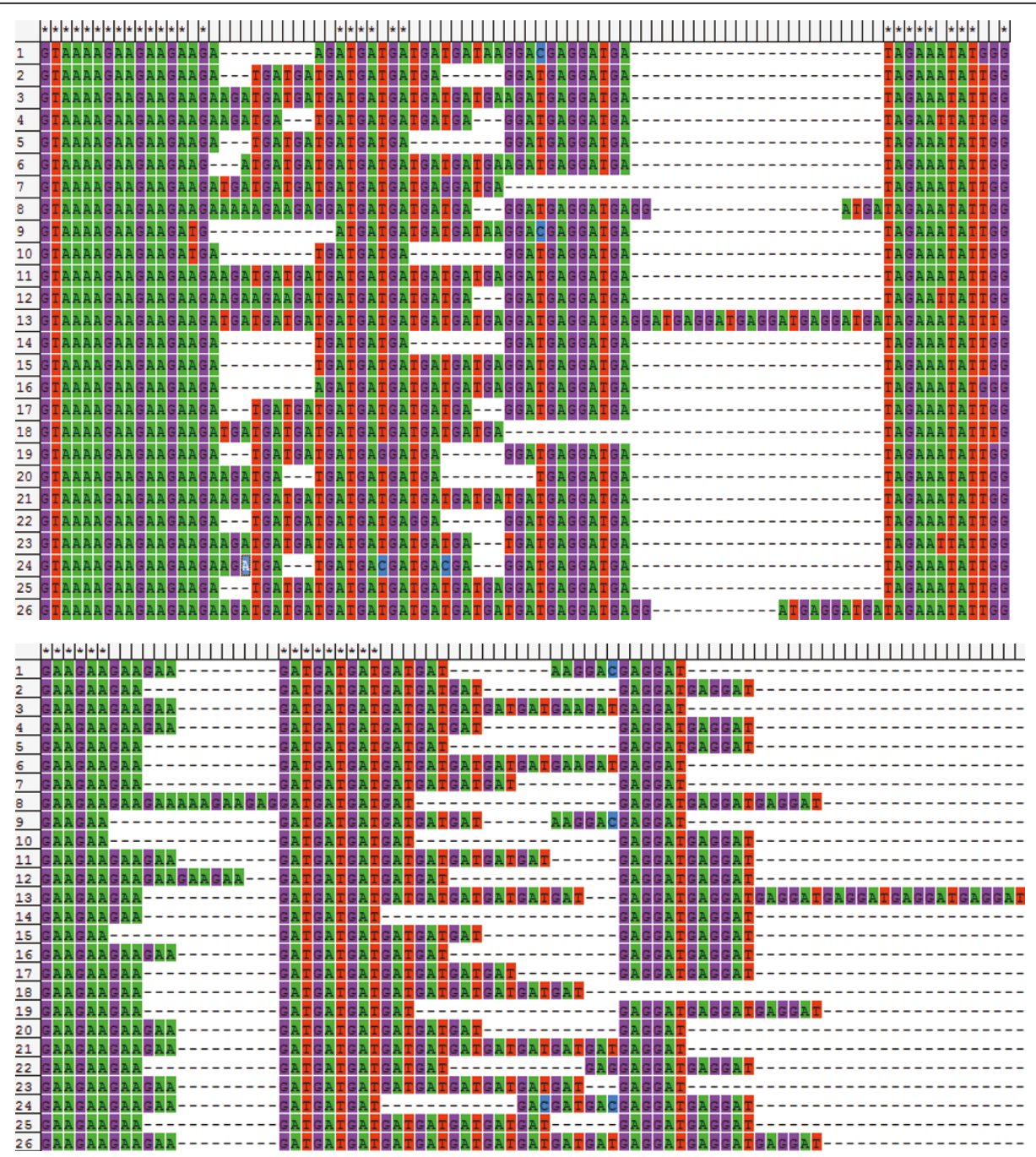

Figure 8 A comparison between two alignments of the sequence of case A by using MEGA4 software (A) and the new software prepared in this paper (B).

1. The first line contains the number of samples, followed by any kind of separator (space or tab...) and, subsequently, the number of nucleotides.

2. Each of the next lines contains the name of the allele, followed by any kind of separator, then the sequence; thereafter press the Enter button to start the next allele.

See the additional file 2: SALT.rar. This is a compressed file containing the program and the sample data used in this research.

\section{Conclusions}

SALT is a new tool to overcome limitations when aligning SSR loci based on the new shifting algorithm proposed in this paper. This tool is essential when aligning compound or imperfect SSRs, which contain many overlaps between repeated units, and when aligning them using the usual methods. The newly developed tool gives a better alignment estimate for such regions.

\section{Materials and methods}

Five different sequences (Table 1) of SSR motifs obtained from a biotechnology laboratory (Genetic Resources Section, ICARDA), were used in this research. These sequences were obtained from 26 plants representing 26 alleles. The sequences were aligned using the clustalW algorithm implemented in MEGA 4 with the following default settings: gap opening penalty 15 , gap extension penalty 6.66, IUB weight matrix, transition weight 0.5 , and delay divergent cut-off 30 [23]. The same software drew the phylogenic tree with the UPGMA method. The PERL programming language was used to design a new algorithm for SSR alignment [24] 

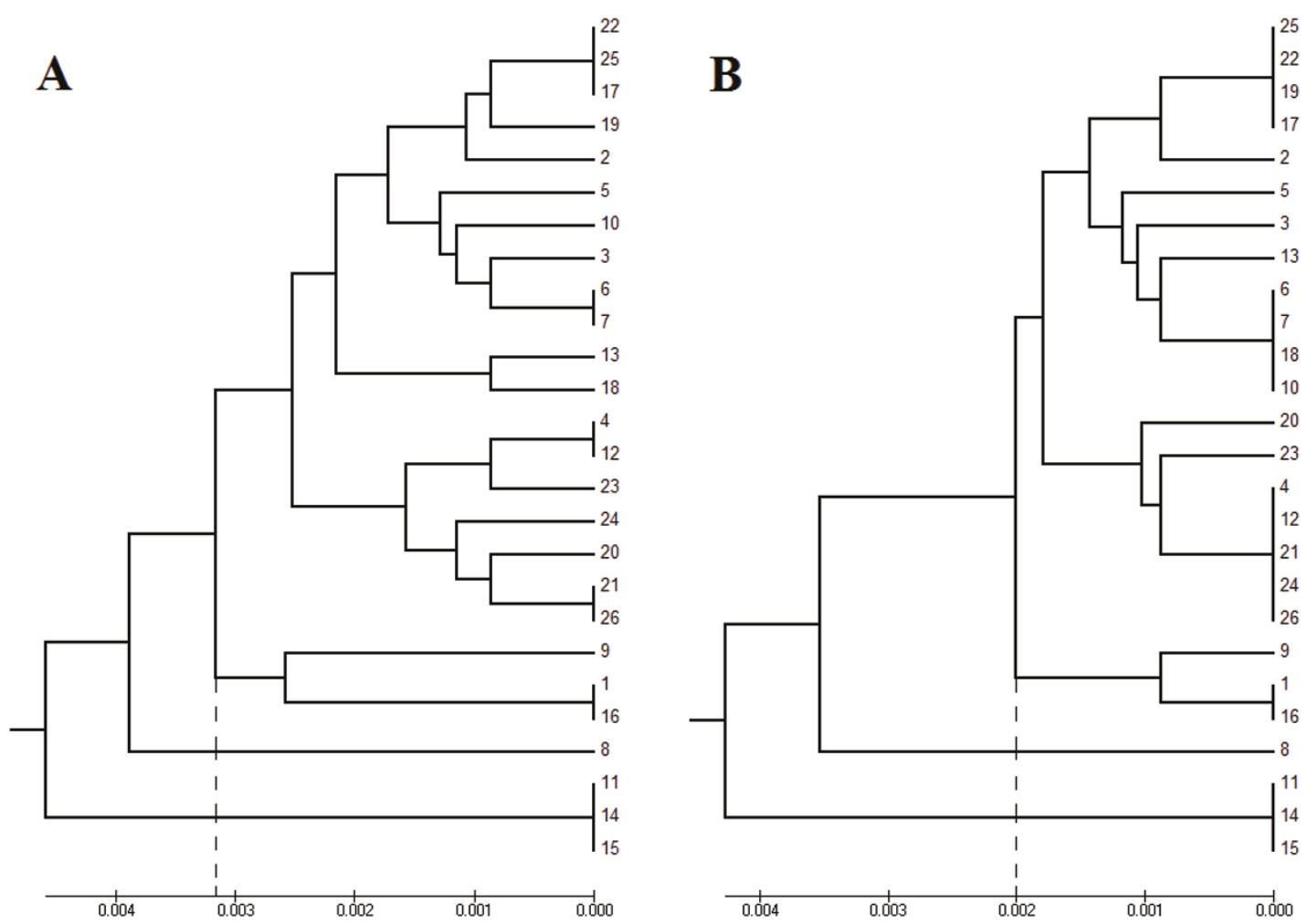

Figure 9 Case E phylogenic tree before (A) and after (B) applying the algorithm.

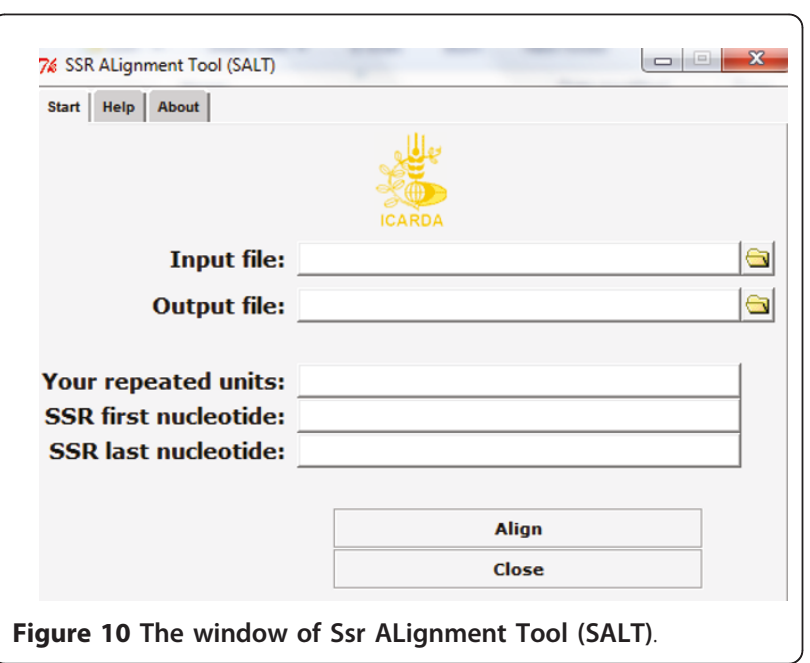

The Tk package was used to make the graphical interface [25].

\section{Additional material}

Additional file 1: An animation describes the algorithm

Additional file 2: A compressed file contains the program (SALT.pl) and the sample data used in this research (the folder: Sample

DATA). (This file could be run with winrar software https://www.win-rar. com)

\section{Authors' contributions}

AJ planned the study, wrote the PERL script, and developed the phylogenic and alignment analysis. AH was involved in the discussion of the analysis.

FCO made substantial contributions towards improving the content and

Table 1 Five microsatellite motifs vary in their types and lengths, representing most SSR types in the genome sequences

\begin{tabular}{llllll}
\hline Case & SSR type & SSR repeat & $\begin{array}{l}\text { Seq. length } \\
\text { (bp) }\end{array}$ & SSR length (bp) & SSR (\%) \\
\hline A & Simple-perfect & $(\mathrm{TA})_{10}$ & 351 & 54 & 15.4 \\
\hline $\mathrm{B}$ & Compound-perfect & $(\mathrm{TA})_{10}(\mathrm{CA})_{16}$ & 397 & 100 & 25.2 \\
\hline $\mathrm{C}$ & Compound-perfect & $(\mathrm{TA})_{10}(\mathrm{CA})_{16}(\mathrm{CG})_{14}$ & 457 & 160 & 35 \\
\hline $\mathrm{D}$ & Compound-perfect & $(\mathrm{TA})_{10}(\mathrm{CA})_{16}(\mathrm{CG})_{14}(\mathrm{TG})_{18}$ & 479 & 182 & 38 \\
\hline $\mathrm{E}$ & Compound-imperfect & $(\mathrm{GAA})_{4}(\mathrm{GAT})_{6}(\mathrm{GAGGAT})_{3}$ & 769 & 72 & 9.4 \\
\hline
\end{tabular}


gave final approval to the version to be published. All the authors drafted the manuscript.

All authors read and approved the final manuscript.

\section{Competing interests}

The authors declare that they have no competing interests.

Received: 18 February 2011 Accepted: 20 July 2011

Published: 20 July 2011

\section{References}

1. Tautz D: Hypervariability of simple sequences as a general source for polymorphic DNA markers. Nucl Acids Res 1989, 17:6563-6571.

2. Peakall R, Gilmore S, Keys W, Morgante M, Rafalski A: Cross-species amplification of soybean (Glycine max) simple sequence repeats (SSRs) within the genus and other legume genera: implications for the transferability of SSRs in plants. Mol Biol Evol 1998, 15:1275-1287.

3. Gow C, Noble JL, Rollinson D, Jones C: A high incidence of clustered microsatellite mutations revealed by parent-offspring analysis in an African freshwater snail, Bulinus forskalii (Gastropoda, Pulmonata). Genetica 2005, 124:77-83.

4. Wiessenbach J, Gyapay G, Dib C, Vignal A, Moresette J: A second generation map of the human genome. Nature 1992, 359:794-801.

5. Weber J, Wong C: Mutation of human short tandem repeats. Hum Mol Genet 1993, 2:1123-1128.

6. Ellegren $\mathrm{H}$ : Microsatellite mutations in the germline: implications for evolutionary inference. Trends Genet 2000, 16:551-558.

7. Chistiakov DA, Hellemans B, Haley CS, Law AS, Tsigenopoulos CS, Kotoulas G, Bertotto D, Libertini A, Volckaert FA: A microsatellite linkage map of the European sea bass Dicentrarchus labrax L. Genetics 2006, 170:1821-1826.

8. Jakupciak JP, Wells RD: Genetic instabilities of triplet repeat sequences by recombination. IUBMB Life 2000, 50:355-359.

9. Ellegren $\mathrm{H}$ : Microsatellites: simple sequences with complex evolution. Nat Rev Genet 2004, 5:435-445.

10. Armour JAL, Alegre SA, Miles S, Williams LJ, Badge RM: Minisatellites and mutation processes in tandemly repetitive DNA. In Microsatellites Evolution and Applications. Edited by: Goldstein DB, Schlötterer C. Oxford: Oxford University Press; 1999:24-33.

11. Kelkar YD, Tyekucheva S, Chiaromonte F, Makova KDL: the genome-wide determinants of human and chimpanzee microsatellite evolution. Genome Res 2008, 18(1):30-38.

12. Whittaker JC, Harbord RM, Boxall N, Mackay I, Dawson G, Sibly RM: Likelihood-based estimation of microsatellite mutation rates. Genetics 2003, 164(2):781-787

13. Sainudiin R, Durrett RT, Aquadro CF, Nielsen R: Microsatellite mutation models: insights from a comparison of humans and chimpanzees. Genetics 2004, 168(1):383-395.

14. Leclercq S, Rivals $E$, Jarne P: DNA slippage occurs at microsatellite loci without minimal threshold length in humans: a comparative genomic approach. Genome Biol Evol 2010, 2:325-335.

15. Zhang Z, Gerstein M: Patterns of nucleotide substitution, insertion and deletion in the human genome inferred from pseudogenes. Nucleic Acids Res 2003, 31:5338-5348.

16. Bell Gl: Evolution of simple sequence repeats. Comput Chem 1996, 20:41-48.

17. Kruglyak S, Durrett R, Schug D, Aquadro C: Equilibrium distributions of microsatellite repeat length resulting from a balance between slippage events and point mutations. Natl Acad Sci USA 1998, 95:10774-10778.

18. Brandström M, Ellegren $\mathrm{H}$ : Genome-wide analysis of microsatellite polymorphism in chicken circumventing the ascertainment bias. Genome Res 2008, 18:881-887.

19. Powell W, Machray GC, Provan J: Polymorphism revealed by simple sequence repeats. Trends Plant Sci 1996, 1:215-222.

20. Kantety RV, Rota ML, Matthews DE, Sorrells ME: Data mining for simple sequence repeats in expressed sequence rags from barley, maize, rice, sorghum and wheat. Plant Molecular Biol 2002, 48:501-510.

21. Santibanez-Koref MF, Gangeswaran R, Hancock JM: A relationship between lengths of microsatellites and nearby substitution rates in mammalian genomes. Mol Biol Evol 2001, 18(11):2119-2123.
22. Sekar M, Suresh E, Kumar NS, Mayak SK, Balakrishna C: Microsatellite DNA markers, a fisheries perspective. Aquaculture Asia Magazine 2009, 27-29.

23. Tamura K, Dudley J, Nei M, Kumar S: MEGA4: molecular evolutionary genetics analysis (MEGA) software version 4.0. Mol Biol Evol 2007, 24:1596-1599.

24. PERL, v5.8.8. , Copyright 1987-2006, Larry Wall. Binary build provided by ActiveState [http://www.ActiveState.com]. The Perl Home Page [http://www. perl.org/].

25. Tk, the extension that makes GUI programming in PERL possible. University of California, Berkeley;[http://www.tcl.tk], Tcl (Tool Command Language) and Tk (ToolKit) was created by Professor John Ousterhout.

doi:10.1186/1756-0500-4-239

Cite this article as: Jighly et al:: Optimization of sequence alignment for simple sequence repeat regions. BMC Research Notes 2011 4:239.

\section{Submit your next manuscript to BioMed Central and take full advantage of:}

- Convenient online submission

- Thorough peer review

- No space constraints or color figure charges

- Immediate publication on acceptance

- Inclusion in PubMed, CAS, Scopus and Google Scholar

- Research which is freely available for redistribution

Submit your manuscript at www.biomedcentral.com/submit
C) Biomed Central 